\title{
RADICAL PERFECTNESS OF PRIME IDEALS IN CERTAIN INTEGRAL DOMAINS
}

\author{
GYU WHAN CHANG AND HWANKOO KIM
}

\begin{abstract}
For a UMT-domain $D$, we characterize when the polynomial ring $D[X]$ is $t$-compactly packed and every prime $t$-ideal of $D[X]$ is radically perfect. As a corollary, for a quasi-Prüfer domain $D$, we also characterize when every prime ideal of $D[X]$ is radically perfect. Finally we introduce the concepts of Serre's conditions in strong Mori domains and characterize Krull domains and almost factorial domains, respectively.
\end{abstract}

Introduction. Let $D$ be an integral domain. In [15], Erdoğdu introduced the notion of radical perfectness of ideals as follows:

An ideal $I$ of $D$ is radically perfect if the height of $I$ is equal to the infimum of the number of generators of ideals of $D$ whose radical is equal to the radical of $I$.

This generalizes the notion of a set-theoretic complete intersection to non-Noetherian rings [16, page 1802]. He then addressed the question of under which conditions all prime ideals of the polynomial ring $D[X]$ over $D$ containing a field of characteristic 0 are radically perfect. In this direction, it was shown [14, Theorem 2.1] that over a Noetherian domain $D$ of Krull dimension 1 containing a field of characteristic 0 , every prime ideal of $D[X]$ is radically perfect if and only if $D$ is a Dedekind domain with torsion ideal class group. In [14, Question 3.3], he also posed the open question:

2010 AMS Mathematics subject classification. Primary 13A15, 13E99, 13F05, $13 \mathrm{G} 05$.

Keywords and phrases. Prüfer domain, radically perfect, $t$-compactly packed, quasi-Prüfer domain, UMT-domain, SM domain, Serre's condition.

The authors were supported by the Basic Science Research Program through the National Research Foundation of Korea (NRF) funded by the Ministry of Education, Science and Technology, grant Nos. 2010-0007069 and 2010-0011996, respectively.

Received by the editors on September 19, 2014, and in revised form on December 26, 2014. 


\begin{abstract}
Suppose that $D$ is an integral domain (not necessarily Noetherian) containing a field of characteristic zero such that each prime ideal of $D[X]$ is radically perfect. Then is $D$ necessarily of dimension one?
\end{abstract}

He answered this question in the positive in the case that $D$ is a Bézout domain [14, Theorem 4.1] or a finite dimensional Prüfer domain of finite character whose maximal ideals are coprimely packed [16, Theorems 3.1, 3.3]. Later, Mimouni also considered this question in different contexts of integral domains, namely, he proved that, for a quasi-Prüfer domain $D$, if every prime ideal of $D[X]$ is radically perfect, then $D$ is a one-dimensional domain and every prime ideal of $D$ is the radical of a principal ideal [30, Theorem 2.1], which is one of Mimouni's main results. He also proved that, for a Prüfer domain $D$, every prime ideal of $D[X]$ is radically perfect if and only if $\operatorname{dim}(D)=1$, every prime ideal of $D$ is the radical of a principal ideal, and the class group $\mathrm{Cl}(D)$ of $D$ is torsion [30, Theorem 2.3].

One purpose of this article is to extend a part of Mimouni's results [30] and to give an answer to an open problem, posed by Erdoğdu and Harman, whether or not height-one prime ideals in $D[X]$ over a Prüfer domain $D$ contracting to zero in $D$ are radically perfect [17], that is, for a UMT-domain $D, D[X]$ is $t$-compactly packed if and only if $D$ is a $t$ compactly packed domain with $\mathrm{Cl}(D[X])$ torsion (Theorem 1.1). As an answer to the problem posed by Erdoğdu and Harman, we show that, for a Prüfer domain $D$, every upper to 0 in $D[X]$ is radically perfect if and only if $\mathrm{Cl}(D)$ is torsion (Corollary 1.7). We also prove that, for a UMT-domain $D$, every prime $t$-ideal of $D[X]$ is radically perfect if and only if $t$ - $\operatorname{dim}(D)=1, \operatorname{Cl}(D[X])$ is torsion, and every prime $t$-ideal of $D$ is radically perfect (Theorem 1.8). As a corollary of this result, it is shown that, if $D$ is a quasi-Prüfer domain, then every prime ideal of $D[X]$ is radically perfect if and only if $\operatorname{dim}(D)=1, \mathrm{Cl}(D[X])$ is torsion, and every prime ideal of $D$ is radically perfect (Corollary 1.10).

For the other purpose, we first recall that $D$ is a strong Mori domain (SM domain) if and only if $D$ is $w$-locally Noetherian and each nonzero element of $D$ lies in only finitely many maximal $w$-ideals $[33$, Theorem 1.9]. This result allows us to extend the concepts of Serre's conditions $\left(R_{n}\right)$ and $\left(S_{n}\right)$ in Noetherian rings to those in SM domains. We prove that an SM domain $D$ satisfies Serre's condition $\left(S_{2}\right)$ if and only if $t$ $\operatorname{dim}(D)=1$ (Proposition 2.1). By using theses notions, we characterize 
Krull domains and almost factorial domains, respectively (Theorem 2.3 and Corollary 2.6).

Let $D$ be an integral domain with quotient field $K, F(D)$ the set of nonzero fractional ideals of $D$ and $f(D)=\{A \in F(D) \mid A$ is finitely generated $\}$; so $f(D) \subseteq F(D)$. For $I \in F(D)$, let $I^{-1}=\{x \in K$ $x I \subseteq D\}, I_{v}=\left(I^{-1}\right)^{-1}, I_{t}=\bigcup\left\{J_{v} \mid J \in f(D)\right.$ with $\left.J \subseteq I\right\}$, and $I_{w}=\left\{x \in K \mid J x \subseteq I\right.$ for some $J \in f(D)$ with $\left.J^{-1}=D\right\}$ [32]. Obviously, $I_{w} \subseteq I_{t} \subseteq I_{v}$ for all $I \in F(D)$. For $*=v, t$ or $w$, an $I \in F(D)$ is called a $*$-ideal if $I_{*}=I$; a $*$-ideal is a maximal $*$-ideal if it is maximal among proper integral $*$-ideals; $*$-Spec $(D)$ denotes the set of prime $*$-ideals; and $*$ - $\operatorname{Max}(D)$ is the set of maximal $*$-ideals. While $v$-Max $(D)$ can be empty, as in the case of $D$ being a rank 1 non-discrete valuation domain, it is well known that $t-\operatorname{Max}(D) \neq \emptyset$ if $D$ is not a field; $t$ - $\operatorname{Max}(D)=w$-Max $(D)$; a maximal $t$-ideal is a prime ideal; every integral $t$-ideal is contained in a maximal $t$-ideal; and $D=\bigcap_{P \in t-\operatorname{Max}(D)} D_{P}$. The $t$-dimension of $D($ denoted by $t$-dim $(D))$ is defined to be the supremum of

$$
\left\{n \mid P_{1} \subsetneq P_{2} \subsetneq \cdots \subsetneq P_{n}, P_{i} \in t-\operatorname{Spec}(D)\right\} .
$$

In particular, if $D$ is not a field, then $t-\operatorname{dim}(D)=1$ if and only if every prime $t$-ideal of $D$ is a maximal $t$-ideal. An $I \in F(D)$ is said to be $t$-invertible if $\left(I I^{-1}\right)_{t}=D$, equivalently, if $I I^{-1} \nsubseteq P$ for every maximal $t$-ideal $P$ of $D$. An integral domain $D$ is called a Prüfer $v$ multiplication domain (PvMD) if every nonzero finitely generated ideal $I$ of $D$ is $t$-invertible, i.e., $\left(I I^{-1}\right)_{t}=D$.

Let $X$ be an indeterminate over $D$, and let $D[X]$ be the polynomial ring over $D$. An upper to zero in $D[X]$ is a nonzero prime ideal $Q$ of $D[X]$ such that $Q \cap D=(0)$. Clearly, if $Q$ is an upper to zero in $D[X]$, then $Q$ is a prime $t$-ideal, ht $Q=1$, and $Q$ is a maximal $t$-ideal if and only if $Q$ is $t$-invertible [25, Theorem 1.4]. We say that $D$ is a UMTdomain if each upper to zero in $D[X]$ is a maximal $t$-ideal of $D[X]$. It is well known that $D$ is an integrally closed UMT-domain if and only if $D$ is a $\mathrm{P} v \mathrm{MD}$ [25, Proposition 3.2] and $D$ is a UMT-domain if and only if $D[X]$ is a UMT-domain, if and only if the integral closure of $D_{P}$ is a Prüfer domain for every maximal $t$-ideal $P$ of $D[\mathbf{1 8}$, Theorems $1.5,2.4]$.

Let $\left\{X_{\alpha}\right\}$ be a nonempty set of indeterminates over $D$. Then $D$ is called a quasi-Prüfer domain if, for each prime ideal $P$ of $D$, if $Q$ is a 
prime ideal of $D\left[\left\{X_{\alpha}\right\}\right]$ with $Q \subseteq P\left[\left\{X_{\alpha}\right\}\right]$, then $Q=(Q \cap D)\left[\left\{X_{\alpha}\right\}\right]$. It is well known that $D$ is quasi-Prüfer if and only if $\bar{D}$, the integral closure of $D$, is a Prüfer domain [19, Corollary 6.5.14], if and only if $D$ is a UMT-domain whose maximal ideals are $t$-ideals [11, Corollary 1.3]. Hence, we have the following implications:

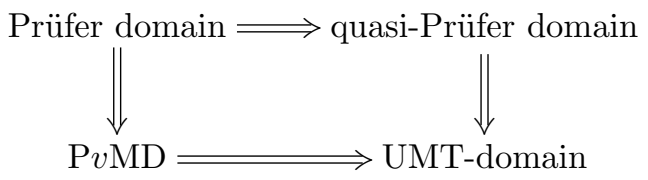

In $[\mathbf{3 2}, \mathbf{3 3}]$, Wang and McCasland defined an integral domain $D$ to be a strong Mori domain (SM domain) if $D$ satisfies the ascending chain condition on integral $w$-ideals. Note that the class of Noetherian domains is properly contained in the class of SM domains, and the class of SM domains is properly contained in the class of Mori domains. (An integral domain $D$ is called a Mori domain if $D$ satisfies the ascending chain condition on integral $v$-ideals.)

Let $T(D)$ be the set of $t$-invertible fractional $t$-ideals of $D$. Then $T(D)$ is an abelian group under the $t$-multiplication $I * J=(I J)_{t}$. Hence, if we let $P(D)$ be the subgroup of nonzero principal fractional ideals of $T(D)$, then $\mathrm{Cl}(D):=T(D) / P(D)$, called the $(t$-) class group of $D$, is an abelian group. Clearly, if $D$ is a Prüfer domain or an integral domain of dimension one (respectively, a Krull domain), then $\mathrm{Cl}(D)$ is the ideal (respectively, divisor) class group of $D$. The notion of ( $t$-)class group was introduced by Bouvier [10], at the suggestion of Zafrullah. We know that a GCD domain $D$ is a $\mathrm{P} v \mathrm{MD}$ with $\mathrm{Cl}(D)=0$ and a UFD $D$ is a Krull domain with $\operatorname{Cl}(D)=0$.

An integral domain $D$ is said to be of finite character (respectively, finite t-character) if each nonzero nonunit of $D$ is contained in only finitely many maximal ideals (respectively, maximal $t$-ideals) of $D$. We know that $D$ is of finite $t$-character if and only if $D[X]$ is of finite $t$ character [22, Lemma 2.1], but if $D$ is not a field, then $D[X]$ is not of finite character because $D[X] / M[X] \cong(D / M)[X]$ for a maximal ideal $M$ of $D$ has infinitely many nonzero prime ideals [3, Proposition 17]. We say that a nonzero element $a \in D$ is a primary element if $a D$ is a primary ideal and that $D$ is a weakly factorial domain (WFD) if each nonzero element of $D$ can be written as a finite product of primary 
elements. Following [27], for $*=t, w$, an integral $*$-ideal $I$ of $D$ is said to be $*$-compactly packed if, for any set $\Lambda$ of prime $*$-ideals of $D$ with $I \subseteq \bigcup_{Q \in \Lambda} Q$, one has $I \subseteq P$ for some $P \in \Lambda$; an integral domain $D$ is said to be $*$-compactly packed if every $*$-ideal of $D$ is $*$-compactly packed.

1. When is every prime ideal of $D[X]$ radically perfect? Let $D$ be an integral domain that is not a field, and let $X$ be an indeterminate over $D$. Recall that $D$ is $t$-compactly packed if and only if every prime $t$-ideal of $D$ is the radical of a principal ideal [12, Proposition 3.1] or [27, Theorem 2.1]. Hence, if $t$-dim $(D)=1$, then $D$ is $t$-compactly packed if and only if each nonzero prime ideal of $D$ contains a primary element [12, Theorem 3.2], if and only if each prime $t$-ideal of $D$ is radically perfect. In this section, we study when $D[X]$ is $t$-compactly packed for a UMT-domain $D$.

Theorem 1.1. If $D$ is a UMT-domain, then $D[X]$ is t-compactly packed if and only if $D$ is a t-compactly packed domain with $\mathrm{Cl}(D[X])$ torsion.

\section{Proof.}

$(\Rightarrow)$. Let $P$ be a prime $t$-ideal of $D$. Then $P[X]$ is a prime $t$-ideal of $D[X]$, and hence, $P[X]=\sqrt{f D[X]}$ for some $f \in P[X]$. Clearly, $f \in D$, and thus, $P=\sqrt{f D}$. Next, let $Q$ be a nonzero prime ideal of $D[X]$ with $Q \cap D=(0)$. Then $Q$ is a maximal $t$-ideal of $D[X]$, and so $Q=\sqrt{g D[X]}$ for some $g \in Q$. Since $Q$ is a maximal $t$-ideal, $g D[X]$ is a primary ideal [2, Lemma 1], and hence, $Q$ contains a primary element. Thus, $\mathrm{Cl}(D[X])$ is torsion [7, Theorem 2.4].

$(\Leftarrow)$. Let $Q$ be a prime $t$-ideal of $D[X]$. If $Q \cap D=(0)$, then $Q$ is a maximal $t$-ideal, and hence, $t$-invertible. Hence, there is an integer $n \geq 1$ such that $\left(Q^{n}\right)_{t}=f D[X]$ for some $f \in Q$. Thus, $Q=\sqrt{f D[X]}$. Next, if $Q \cap D \neq(0)$, then $Q \cap D$ is a prime $t$-ideal of $D$ and $Q=(Q \cap D)[X]$ because $D$ is a UMT-domain. Hence, $Q \cap D=\sqrt{a D}$ for some $a \in D$. Thus, $Q=\sqrt{a D[X]}$.

Corollary 1.2. If $D$ is a $\mathrm{P} v \mathrm{MD}$, then $D[X]$ is t-compactly packed if and only if $D$ is a t-compactly packed domain with $\mathrm{Cl}(D)$ torsion. 
Proof. This is an immediate consequence of Theorem 1.1 because

(i) $D$ is a $\mathrm{P} v \mathrm{MD}$ if and only if $D$ is an integrally closed UMT-domain, and

(ii) $\mathrm{Cl}(D[X])=\mathrm{Cl}(D)$ if and only if $D$ is integrally closed [21, Theorem 3.6].

An integral domain $D$ is called an almost GCD domain (AGCD domain) if, for each $0 \neq a, b \in D$, there is an integer $n=n(a, b) \geq 1$ such that $a^{n} D \cap b^{n} D$ is principal. Clearly, a GCD domain is an integrally closed AGCD domain, although there do exist integrally closed AGCD domains such as almost factorial domains as characterized in [20, Proposition 6.8]. Also, it is known that, if $D$ is integrally closed, then $D$ is an AGCD domain if and only if $D$ is a $\mathrm{P} v \mathrm{MD}$ with $\mathrm{Cl}(D)$ torsion [34, Theorem 3.9]. Hence, by Corollary 1.2, we have

Corollary 1.3. If $D$ is an integrally closed AGCD-domain, then $D$ is $t$-compactly packed if and only if $D[X]$ is t-compactly packed.

The next results are already known, but we use our results to give simple proofs. For convenience, let $X^{(1)}(D)$ be the set of height 1 prime ideals of $D$.

Corollary 1.4. Let $D$ be a Krull domain, and hence, $t-\operatorname{dim}(D)=1$.

(1) (cf., [9, Proposition 3.1]). The following statements are equivalent.

(a) $D$ is t-compactly packed.

(b) $\mathrm{Cl}(D)$ is torsion.

(c) $D[X]$ is t-compactly packed.

(d) Each prime t-ideal of $D$ is radically perfect.

(2) $[\mathbf{1 7}$, Theorem 4.1]. If $D$ is a two-dimensional domain of finite character, then each prime ideal of $D$ is radically perfect if and only if $\mathrm{Cl}(D)$ is torsion.

Proof.

(1) (a) $\Leftrightarrow(\mathrm{b})$. Since $D$ is a Krull domain, $\mathrm{Cl}(D)$ is torsion if and only if each $P \in X^{(1)}(D)$ is the radical of a principal ideal. Thus, as each prime $t$-ideal is in $X^{(1)}(D), D$ is $t$-compactly packed if and only if $\mathrm{Cl}(D)$ is torsion. 
(b) $\Rightarrow(\mathrm{c})$. Note that a Krull domain is a $\mathrm{P} v \mathrm{MD}$ and $D$ is $t$-compactly packed by (a). Hence, by Corollary $1.2, D[X]$ is $t$ compactly packed.

$(\mathrm{c}) \Rightarrow(\mathrm{a})$. This follows from Theorem 1.1 because a Krull domain is a UMT-domain.

(a) $\Leftrightarrow(\mathrm{d})$. This follows because $t$ - $\operatorname{dim}(D)=1$.

(2) $(\Rightarrow)$. This is an immediate consequence of (1) above.

$(\Leftarrow)$. Let $M$ be a nonzero prime ideal of $D$. If $\operatorname{ht}(M)=1$, then $M$ is a $t$-ideal, and hence, by $(1), M$ is radically perfect. Next, assume $\operatorname{ht}(M)=2$. Choose $0 \neq a \in M$. Since $D$ is of finite character, there are only finitely many maximal ideals $M, M_{1}, \ldots, M_{k}$ of $D$ containing $a$. Also, since $D$ is a Krull domain, there do exist only finitely many height 1 prime ideals, say $P_{1}, \ldots, P_{n}$, that contain $a$. Hence, we can choose another $b \in M \backslash\left(\left(\bigcup M_{i}\right) \cup\left(\bigcup P_{i}\right)\right)$, and thus, $M=\sqrt{(a, b)}$. If $M=\sqrt{x D}$ for some $x \in D$, then $M$ is minimal over $x D$, and hence, $M$ is a $t$-ideal, which is contrary to the fact that $t-\operatorname{dim}(D)=1$. Thus, $M$ is radically perfect.

Let $Q$ be an upper to zero in $D[X]$. Then $\operatorname{ht}(Q)=1$, and hence, $Q$ is radically perfect if and only if $Q=\sqrt{f D[X]}$ for some $f \in Q$. Hence, in this case, $Q$ is a maximal $t$-ideal if and only if $f$ is a primary element [2, Lemma 1]. Thus, by [7, Theorem 2.4], we have

Lemma 1.5. If $D$ is a UMT-domain, then every upper to zero in $D[X]$ is radically perfect if and only if $\mathrm{Cl}(D[X])$ is torsion.

Clearly, if $D$ is a GCD domain, then $D$ is a UMT-domain and $\mathrm{Cl}(D[X])=\mathrm{Cl}(D)=0$. Thus, by Lemma 1.5 , every upper to zero in $D[X]$ over a GCD domain $D$ is radically perfect. More generally, we have

Corollary 1.6. If $D$ is an AGCD domain, then every upper to zero in $D[X]$ is radically perfect if and only if $\mathrm{Cl}(D[X])$ is torsion.

Proof. This follows directly from Lemma 1.5 because an AGCD domain is a UMT-domain [7, Lemma 3.1].

In [17, page 539], the authors say "We failed in our attempt to see whether or not height-one prime ideals in $D[X]$ over a Prüfer domain $D$ 
contracting to zero in $D$ are radically perfect." Note that height 1 prime ideals in $D[X]$ contracting to 0 in $D$ are just the uppers to zero in $D[X]$. Hence, the next result is an answer to the open problem in $[\mathbf{1 7}$, page $539]$.

Corollary 1.7. If $D$ is a Prüfer domain, then every upper to zero in $D[X]$ is radically perfect if and only if $\mathrm{Cl}(D)$ is torsion.

Proof. This is an immediate consequence of Lemma 1.5 because Prüfer domains are UMT-domains and $\mathrm{Cl}(D)=\mathrm{Cl}(D[X])$ when $D$ is integrally closed.

An integral domain $D$ is called a weakly Krull domain if

$$
D=\bigcap_{P \in X^{(1)}(D)} D_{P}
$$

and this intersection is locally finite. Hence, $D$ is a weakly Krull domain if and only if $t$ - $\operatorname{dim}(D)=1$ and $D$ is of finite $t$-character. An almost WFD (AWFD) is a weakly Krull domain with torsion $(t-)$ class group; and $D$ is a generalized WFD (GWFD) if each nonzero prime ideal of $D$ contains a primary element. It is known that $\mathrm{WFD} \Rightarrow \mathrm{AWFD} \Rightarrow$ GWFD $\Rightarrow$ weakly Krull domain. Recall from $[30$, Lemma 3.4] that, if every prime ideal of $D[X]$, where $D$ is a UMT-domain, is radically perfect, then $t$ - $\operatorname{dim}(D)=1$.

Theorem 1.8. The following statements are equivalent for a UMTdomain $D$.

(1) Every prime t-ideal of $D[X]$ is radically perfect.

(2) $t-\operatorname{dim}(D)=1, \operatorname{Cl}(D[X])$ is torsion and every prime $t$-ideal of $D$ is radically perfect.

(3) $t-\operatorname{dim}(D)=1, \operatorname{Cl}(D[X])$ is torsion and $D$ is of finite $t$-character.

(4) $D$ is a weakly Krull domain and $\mathrm{Cl}(D[X])$ is torsion.

(5) $D[X]$ is an AWFD.

(6) $D[X]$ is a GWFD.

Proof.

$(1) \Rightarrow(2)$. For $t$-dim $(D)=1$, see the proof of [30, Lemma 3.4]. Next, note that an upper to zero in $D[X]$ is a prime $t$-ideal; hence, by 
Lemma 1.5, $\mathrm{Cl}(D[X])$ is torsion. Finally, for the radical perfectness of every prime $t$-ideal of $D$, let $P$ be a prime $t$-ideal of $D$. Then $P[X]$ is a prime $t$-ideal of $D[X]$ and $h t(P[X])=h t(P)=1$ because $D$ is a UMT-domain and $t$-dim $(D)=1$. Hence, $P[X]=\sqrt{g D[X]}$ for some $g \in P[X]$, and since $P[X] \cap D \neq(0), g$ must be in $P$. Thus, $P=\sqrt{g D}$.

$(2) \Rightarrow(3)$. Let $a \in D$ be a nonzero nonunit. Then each prime ideal of $D$ minimal over $a D$ is a maximal $t$-ideal and the radical of a principal ideal by assumption. Hence, the number of maximal $t$-ideals of $D$ containing $a$ is finite [24, Theorem 1.6].

$(3) \Leftrightarrow(4)$. Clear.

$(4) \Leftrightarrow(5)$. This is an immediate consequence of the fact that $D$ is a weakly Krull UMT-domain if and only if $D[X]$ is a weakly Krull domain [4, Proposition 4.11].

$(5) \Leftrightarrow(6)$. [7, Corollary 2.9].

$(6) \Rightarrow(1)$. We use the equivalence of $(3)-(6)$ above. Let $Q$ be a prime $t$-ideal of $D[X]$. Then $h t(Q)=1$ because $t$-dim $(D[X])=1$, and hence, either $Q \cap D=(0)$ or $Q=(Q \cap D)[X]$ and $Q \cap D$ is a $t$-ideal of $D$ [25, Proposition 1.1]. If $Q \cap D=(0)$, then $Q$ is $t$-invertible because $D$ is a UMT-domain. Hence, as $\mathrm{Cl}(D[X])$ is torsion, $\left(Q^{n}\right)_{t}=f D[X]$ for some integer $n \geq 1$ and $f \in Q$, and thus, $Q=\sqrt{f D[X]}$. If $Q=(Q \cap D)[X]$, then $Q \cap D=\sqrt{b D}$ for some $b \in D$ because $D$ is a GWFD [8, Proposition 2.3], and so, $Q=\sqrt{b D[X]}$. Therefore, every prime $t$-ideal of $D[X]$ is radically perfect.

Corollary 1.9. The following statements are equivalent for $a \mathrm{P} v \mathrm{MD} D$.

(1) Every prime $t$-ideal of $D[X]$ is radically perfect.

(2) $t-\operatorname{dim}(D)=1, \operatorname{Cl}(D)$ is torsion, and every prime $t$-ideal of $D$ is radically perfect.

(3) $t-\operatorname{dim}(D)=1, \operatorname{Cl}(D)$ is torsion, and $D$ is of finite $t$-character.

(4) $D$ is an AWFD.

(5) $D[X]$ is an AWFD.

(6) $D[X]$ is a GWFD.

Proof. This is an immediate consequence of Theorem 1.8 since a $\mathrm{P} v \mathrm{MD} D$ is an integrally closed UMT-domain and $\mathrm{Cl}(D)=\mathrm{Cl}(D[X])$. 
Let $D$ be a quasi-Prüfer domain. In [30, Theorem 2.1], Mimouni showed that, if every prime ideal of $D[X]$ is radically perfect, then $D$ is a one-dimensional domain and every prime ideal of $D$ is the radical of a principal ideal.

We next give a complete characterization of a quasi-Prüfer domain $D$ such that every prime ideal of $D[X]$ is radically perfect. To do this, it is useful to recall that $D$ is a quasi-Prüfer domain if and only if $D$ is a UMT-domain whose maximal ideals are $t$-ideals [11, Corollary 1.3]. Also, note that, if $P$ is a prime ideal of $D$ with $\operatorname{ht}(P)=1$, then $P$ is radically perfect if and only if $P$ is the radical of a principal ideal.

Corollary 1.10. The following statements are equivalent for a quasiPrüfer domain D.

(1) Every prime ideal of $D[X]$ is radically perfect.

(2) Every prime $t$-ideal of $D[X]$ is radically perfect.

(3) $\operatorname{dim}(D)=1, \operatorname{Cl}(D[X])$ is torsion, and every prime ideal of $D$ is radically perfect.

Proof.

$(1) \Rightarrow(2)$. Clear.

$(2) \Rightarrow(3)$. This follows directly from Theorem 1.8 because a quasiPrüfer domain is a UMT-domain whose maximal ideals are $t$-ideals.

$(3) \Rightarrow(1)$. Let $\bar{D}$ be the integral closure of $D$. Then $\bar{D}$ is a Prüfer domain [19, Corollary 6.5.14] and $\bar{D}[X]=\overline{D[X]}$; so $\operatorname{dim}(D[X])=$ $\operatorname{dim}(\bar{D}[X])=2$ [23, Proposition 30.4].

Next, let $Q$ be a nonzero prime ideal of $D[X]$. If $\operatorname{ht}(Q)=1$, then $Q=\sqrt{f D[X]}$ for some $f \in Q$ by the proof of Theorem 1.8 $(6) \Rightarrow(1)$. Assume $\operatorname{ht}(Q)=2$. Then $Q \neq \sqrt{g D[X]}$ for all $g \in D[X]$ because $Q$ is not a $t$-ideal, and $(0) \subsetneq(Q \cap D)[X] \subsetneq Q$. Hence, $Q /(Q \cap D)[X]$ is a nonzero prime ideal of $D[X] /(Q \cap D)[X]$ and $D[X] /(Q \cap D)[X] \cong(D / Q \cap D)[X]$ is a PID. So there is an $h \in Q$ such that $Q /(Q \cap D)[X]=(h D[X]+(Q \cap D)[X]) /(Q \cap D)[X]$. Note that $Q \cap D=\sqrt{b D}$ for some $b \in Q \cap D$. Thus, $Q=\sqrt{(h, b) D[X]}$.

It is known that $D[X]$ is a WFD if and only if $D$ is a weakly factorial GCD domain [5, Theorem 17]. Hence, by Corollary 1.9, every prime $t$-ideal of a WFD $D[X]$ is radically perfect. 
The next result is already known [30, Theorem 2.3], but we can recover it easily.

Corollary 1.11. The following statements are equivalent for a Prüfer domain $D$.

(1) Every prime ideal of $D[X]$ is radically perfect.

(2) $\operatorname{dim}(D)=1, \mathrm{Cl}(D)$ is torsion and every prime ideal of $D$ is radically perfect.

(3) $\operatorname{dim}(D)=1, \mathrm{Cl}(D)$ is torsion and $D$ is of finite character.

Proof. This is an immediate consequence of Corollaries 1.9 and 1.10 because a Prüfer domain is a $\mathrm{P} v \mathrm{MD}$ whose maximal ideals are $t$ ideals.

Corollary 1.12. The following statements are equivalent for a Dedekind domain $D$.

(1) Every prime ideal of $D[X]$ is radically perfect.

(2) $\mathrm{Cl}(D)$ is torsion.

(3) Every prime ideal of $D$ is radically perfect.

Proof.

$(1) \Leftrightarrow(2) \Rightarrow(3)$. This is an immediate consequence of Corollary 1.11 because a Dedekind domain is a one-dimensional Prüfer domain of finite character.

$(3) \Rightarrow(2)$. Since a Dedekind domain is a Krull domain, $\mathrm{Cl}(D)$ is torsion by Corollary 1.4 (1).

2. On strong Mori domains satisfying Serre's conditions. Let $R$ be a Noetherian ring. Recall the Serre's conditions $\left(R_{i}\right)$ and $\left(S_{i}\right)$ for $i=0,1,2, \ldots$.

$\left(R_{i}\right) R_{P}$ is regular for all prime ideals $P$ of $R$ with $h t(P) \leq i$, and

$\left(S_{i}\right) \operatorname{depth}\left(R_{P}\right) \geq \min \{\operatorname{ht}(P), i\}$ for all prime ideals $P$ of $R$.

The Krull-Serre normality criterion says that a Noetherian domain $D$ is integrally closed if and only if $\left(R_{1}\right)$ and $\left(S_{2}\right)$ hold in $D[\mathbf{2 9}$, Theorem 23.8]. 
Now we extend the concepts $\left(R_{i}\right)$ and $\left(S_{i}\right)$ in Noetherian domains to those in SM domains. Let $D$ be an SM domain and $n$ a positive integer. Then we say that $D$ satisfies Serre's condition $\left(R_{n}\right)$ if, for any $P \in w$-Spec $(D)$ of height $m \leq n$, the localization $D_{P}$ is regular of dimension $m$. We also say that $D$ satisfies Serre's condition $\left(S_{n}\right)$ if $\operatorname{depth}\left(D_{P}\right) \geq \min \{n, \operatorname{ht}(P)\}$ for all $P \in w$-Spec $(D)$. Clearly, if an SM domain $D$ satisfies $\left(R_{n}\right)$ (respectively, $\left(S_{n}\right)$ ), then $D_{P}$ as a Noetherian domain satisfies $\left(R_{n}\right)$ (respectively, $\left.\left(S_{n}\right)\right)$ for all $P \in w$-Spec $(D)$. Set $\Delta:=\left\{P \in w-\operatorname{Spec}(D) \mid \operatorname{depth}\left(D_{P}\right)=1\right\}$. Then $X^{(1)}(D) \subseteq \Delta$ and

$$
D=\bigcap_{P \in \Delta} D_{P} .
$$

Let $D$ be any integral domain. Following [31], we say that $D$ satisfies $\left(S_{2}^{*}\right)$ if every nonzero principal ideal $x D$ is expressed as a finite intersection $Q_{1} \cap \cdots \cap Q_{n}$ of primary $w$-ideals $Q_{i}$ with $\sqrt{Q_{i}} \in X^{(1)}(D)$. If $D$ satisfies $\left(S_{2}^{*}\right)$, then, for a prime ideal $P$ with ht $(P) \geq 2, P$ contains $a, b \in D$ such that $a, b$ is a $D_{P}$-sequence, and hence,

$$
D=\bigcap_{P \in X^{(1)}} D_{P} .
$$

Note that $D$ satisfies $\left(S_{2}^{*}\right)$ if and only if $D$ is a weakly Krull domain [6, Theorem 3.1].

It is well known that, for a Noetherian local ring $R$ with maximal ideal $\mathfrak{m}$ and a nonzero finitely generated $R$-module $M, \operatorname{depth}(M)=1$ if and only if there is an $x \in \mathfrak{m}$ such that $x$ is not a zero-divisor of $M$ and $\mathfrak{m}$ is an associated prime ideal of $M / x M$.

Proposition 2.1 (cf., [1, Lemma 23.16]). The following statements are equivalent for an $\mathrm{SM}$ domain $D$.

(1) D satisfies $\left(S_{2}\right)$.

(2) Every prime divisor of a nonzero principal ideal of $D$ has height 1.

(3) For all $P \in w-\operatorname{Spec}(D), P D_{P}$ contains a regular sequence of length at least $\min \{2, \operatorname{ht}(P)\}$.

(4) $D$ satisfies $\left(S_{2}^{*}\right)$.

(5) $X^{(1)}(D)=\Delta$.

(6) $t-\operatorname{dim}(D)=1$. 
Proof.

$(1) \Rightarrow(2)$. It is well known that, for a Noetherian domain, $\left(S_{2}\right)$ is equivalent to the condition that every prime divisor of a nonzero principal ideal has height 1 [29, page 183].

Now let $P$ be a prime divisor of a nonzero principal ideal $(a)$ of $D$. Then $P=(a D: b)$ for some $b \in D$. Then $P$ is a prime $w$-ideal. Thus, $P$ is contained in some maximal $w$-ideal $M$ of $D$. By [33, Theorem 1.9], $D_{M}$ is Noetherian and, since $P_{M}$ is a prime divisor of $a D_{M}$, we have ht $(P)=\operatorname{ht}\left(P_{M}\right)=1$.

$(2) \Rightarrow(5)$. If $P \in \Delta$, then $\operatorname{depth}\left(D_{P}\right)=1$. Then, by the above remark, $P D_{P}$ is a prime divisor of a nonzero principal ideal of $D_{P}$. It is routine to show that $P$ is a prime divisor of a nonzero principal ideal of $D$. Thus by hypothesis, $P$ has height 1 .

$(5) \Rightarrow(6)$. Suppose on the contrary that $t$-dim $(D) \geq 2$. Then there exists a prime $t$-ideal $P$ with $\operatorname{ht}(P) \geq 2$. Since $D$ is an $\mathrm{SM}$ domain, $P D_{P}$ is a prime $t$-ideal of $D_{P}$, [35]. But, by hypothesis, $P D_{P}$ contains a regular sequence of length 2. Thus, by [26, Exercise 1, p.102], $\left(P D_{P}\right)^{-1}=D_{P}$, which is a contradiction.

$(6) \Rightarrow(4)$. This is clear.

$(4) \Rightarrow(3)$. Suppose that $D$ satisfies $\left(S_{2}^{*}\right)$. Then, by [31, Remark (3)], for a prime ideal $P$ with $h t(P) \geq 2, P$ contains elements $f, g \in D$ such that $f, g$ is a $D_{P}$-regular sequence. $\left(S_{2}\right)$.

$(3) \Rightarrow(1)$. This follows from the definition of Serre's condition

Recall from [28] that $D$ is an infra-Krull domain if:

(i) $D=\bigcap_{P \in X^{(1)}(D)} D_{P}$, where the intersection is locally finite, and

(ii) $D_{P}$ is Noetherian for each $P \in X^{(1)}(D)$.

Thus, $D$ is an infra-Krull domain if and only if $D$ is an SM domain with $t-\operatorname{dim}(D)=1$. We also remark that several equivalent conditions for an SM domain $D$ to be $t$ - $\operatorname{dim}(D)=1$ are given in [13, Corollary 3.2]. In particular, an SM domain is of $t$-dimension 1 if and only if it is a UMT-domain. Hence, an SM domain satisfies $\left(S_{2}\right)$ if and only if it is a UMT-domain, if and only if it is a weakly Krull domain. 
Combining Proposition 2.1 with [27, Theorem 3.6], we obtain the next result.

Corollary 2.2. Let $D$ be an $\mathrm{SM}$ domain satisfying the condition $\left(S_{2}\right)$. Then the following statements are equivalent.

(1) $D$ is w-compactly packed.

(2) $D$ is t-compactly packed.

(3) Every t-ideal of $D$ is radically perfect.

(4) Every $w$-ideal of $D$ is radically perfect.

(5) Every (t-)subintersection $\bigcap_{P \in \Lambda} D_{P}(\Lambda \subseteq t$-Spec $(D))$ is a ring of quotients of $D$.

Now we can characterize Krull domains in terms of Serre's conditions $\left(S_{2}\right)$ and $\left(R_{1}\right)$ over SM domains. The equivalence of (1) and (4) is already shown in [6, Corollary 3.2] without the assumption that $D$ is an SM domain.

Theorem 2.3. The following statements are equivalent for an SM domain D.

(1) $D$ is a Krull domain.

(2) D satisfies $\left(S_{2}\right)$ and $\left(R_{1}\right)$.

(3) $D$ satisfies $\left(S_{2}\right)$, and, for every $P \in X^{(1)}(D)$, any $P$-primary $w$ ideal $Q$ is a symbolic power, i.e., $Q=P^{(n)}$ for some $n \in \mathbb{N}$.

(4) For any nonzero nonunit $d \in D, d D$ is expressed by a finite intersection of symbolic powers of prime ideals in $X^{(1)}(D)$.

Proof.

$(1) \Rightarrow(3)$. This is obvious.

$(3) \Rightarrow(2)$. It suffices to show that $D_{P}$ is a DVR for each $P \in$ $X^{(1)}(D)$. So we may assume that $(D, P)$ is a one-dimensional local Noetherian domain satisfying the condition that every $P$-primary ideal is of the form $P^{k}$ for some $k \in \mathbb{N}$. Now note that every nonzero proper ideal of $D$ is $P$-primary. Thus, $D$ is a DVR.

$(2) \Rightarrow(1)$. Assume that $D$ satisfies $\left(S_{2}\right)$ and $\left(R_{1}\right)$. Then it suffices to show that $D$ is integrally closed, since $D$ is a Krull domain if and only if $D$ is an integrally closed SM domain. Note that $t-\operatorname{dim}(D)=1$ 
by Proposition 2.1. So $D=\bigcap_{P \in X^{(1)}(D)} D_{P}$. Also, $D_{P}$ is a DVR, and hence, $D_{P}$ is integrally closed for each $P \in X^{(1)}(D)$. Thus, $D$ is integrally closed.

$(1) \Rightarrow(4)$. This is obvious.

$(4) \Rightarrow(1)$. Clearly, $D$ satisfies $\left(S_{2}^{*}\right)$, and so, $t$-dim $(D)=1$ by Proposition 2.1. Also, if $P \in X^{(1)}(D)$, then, for any $0 \neq d \in P$, we have $d D_{P}=P^{(n)} D_{P}=P^{n} D_{P}=\left(P D_{P}\right)^{n}$ for some integer $n \geq 1$. Hence, $P D_{P}$ is invertible, and so principal. Thus, $D_{P}$ is a DVR.

It was shown in [12, Theorem 3.2] that an integral domain $D$ with $t$ - $\operatorname{dim}(D)=1$ is a GWFD if and only if $D$ is $t$-compactly packed. Note that it was shown in [31, Lemma 17] that, for a Noetherian domain $D$, if every $P \in X^{(1)}(D)$ contains a nonzero primary element, then $D$ satisfies $\left(S_{2}\right)$. The next result is a variant of these results.

Lemma 2.4. The following statements are equivalent for an SM domain $D$.

(1) $D$ is a GWFD.

(2) D is t-compactly packed.

(3) Every $P \in X^{(1)}(D)$ contains a nonzero primary element.

Proof.

$(1) \Rightarrow(3)$. Clear.

$(3) \Rightarrow(2)$. Let $Q$ be a prime $t$-ideal of $D$. Since $D$ is an SM domain, $Q$ contains a prime ideal $P \in X^{(1)}(D)$. By assumption, $P$ contains a nonzero primary element $x$, and since $\sqrt{x D}$ is a maximal $t$-ideal [8, Lemma 2.1], $Q=P=\sqrt{x D}$. Thus, $D$ is $t$-compactly packed.

$(2) \Rightarrow(1)$. Let $P$ be a maximal $t$-ideal of $D$ such that $P=\sqrt{a D}$ for some $a \in D$. Then $P$ is minimal over $a D$, and hence $\operatorname{ht}(P)=1[\mathbf{3 3}$, Corollary 1.11]. Thus, $t-\operatorname{dim}(D)=1$. Also, since $\sqrt{a D}$ is a maximal $t$-ideal, $a D$ is primary [8, Lemma 2.1], and thus, $P$ contains a primary element $a$. Thus, $D$ is a GWFD.

The next lemma is due to Storch [20, page 34] under the hypothesis that $D$ is Noetherian. The proof works also for SM domains along with Lemma 2.4. 
Lemma 2.5. Let $D$ be an $S M$ domain. Assume that, for each $P \in$ $X^{(1)}(D), P^{(n)}\left(:=P^{n} D_{P} \cap D\right)$ is principal for some $n \in \mathbb{N}$. Then $D$ is integrally closed and so an almost factorial domain.

An almost factorial domain $D$ is exactly a Krull domain with $\mathrm{Cl}(D)$ torsion. In [31, Proposition 7] and [8, Proposition 3.1], it is shown that a Krull domain $D$ is almost factorial if and only if every $P \in X^{(1)}(D)$ is radically perfect, if and only if every $P \in X^{(1)}(D)$ contains a nonzero primary element, if and only if $D$ is a GWFD. An overring $R$ of an integral domain $D$ is said to be $t$-linked over $D$ if $I^{-1}=D$ for $I \in f(D)$ implies $(I R)_{v}=R$. Recall that $D$ is a $t \mathrm{QR}$ domain if each $t$-linked overring of $D$ is a quotient ring of $D$.

Corollary 2.6. The following statements are equivalent for an SM domain $D$.

(1) $D$ is almost factorial.

(2) For every $P \in X^{(1)}(D), P^{(n)}$ is principal for some $n \in \mathbb{N}$.

(3) For each $P \in X^{(1)}(D)$, any $P$-primary $w$-ideal is a symbolic power, and $P$ contains a nonzero primary element in $D$.

(4) $D$ is integrally closed and any $P \in X^{(1)}(D)$ is radically perfect.

(5) $D$ is a $t \mathrm{QR}$ domain.

Proof.

$(1) \Rightarrow(3)$. Let $P \in X^{(1)}(D)$. Then, since an almost factorial domain is a Krull domain, by Theorem 2.3, any $P$-primary $w$-ideal is of a form $P^{(n)}$ for some integer $n \geq 1$. Also, since $\mathrm{Cl}(D)$ is torsion, there is an integer $k \geq 1$ such that $\left(P^{k}\right)_{t}=x D$ for some $x \in P$. Note that $P$ is a maximal $t$-ideal; so $x D$ is primary [8, Lemma 2.1]. Thus, $P$ contains a nonzero primary element.

$(3) \Rightarrow(2)$. Clear.

$(2) \Rightarrow(1)$. Lemma 2.5 .

$(1) \Leftrightarrow(4)$. This follows from [31, Proposition 7] because an integrally closed SM domain is a Krull domain.

$(1) \Leftrightarrow(5)$. [27, Theorem 3.1]. 
Acknowledgments. We would like to thank the referee for several valuable comments and suggestions, which resulted in an improved version of the paper.

\section{REFERENCES}

1. A. Altman and S. Kleiman, A term of commutative algebra, http://web.mit. edu/18.705/www/12Nts-2up.pdf.

2. D.D. Anderson, D.F. Anderson and M. Zafrullah, Atomic domains in which almost all atoms are prime, Comm. Algebra 20 (1992), 1448-1462.

3. D.D. Anderson, G.W. Chang and M. Zafrullah, Nagata-like theorems for integral domains of finite character and finite t-character, J. Alg. Appl. 14 (2015), 1550119.

4. D.D. Anderson, E.G. Houston and M. Zafrullah, t-linked extensions, the $t$ class group, and Nagata's theorem, J. Pure Appl. Algebra 86 (1993), 109-124.

5. D.D. Anderson and L.A. Mahaney, On primary factorizations, J. Pure Appl. Algebra 54 (1988), 141-154.

6. D.D. Anderson, J.L. Mott and M. Zafrullah, Finite character representations for integral domains, Boll. U.M.I. 6 (1992), 613-630.

7. D.F. Anderson and G.W. Chang, Almost splitting sets in integral domains, II, J. Pure Appl. Algebra 208 (2007), 351-359.

8. D.F. Anderson, G.W. Chang and J. Park, Generalized weakly factorial domains, Houston J. Math. 29 (2003), 1-13.

9. A. Benobaid and A. Mimouni, Compact and coprime packedness with respect to star operations, Houston J. Math. 37 (2011), 1043-1061.

10. A. Bouvier, Le groupe des classes d'un anneau intégré, Soc. Savantes, Brest 4 (1982), 85-92.

11. G.W. Chang and M. Fontana, Uppers to zero in polynomial rings and Prüfer-like domains, Comm. Algebra 37 (2009), 164-192.

12. G.W. Chang and C.J. Hwang, Coverring and intersection conditions for prime ideals, Korean J. Math. 17 (2009), 15-23.

13. G.W. Chang and M. Zafrullah, The w-integral closure of integral domains, J. Algebra 295 (2006), 195-210.

14. V. Erdoğdu, Coprime packedness and set theoretic complete intersection of ideals in polynomial rings, Proc. Amer. Math. Soc. 132 (2004), 3467-3471.

15. , Radically perfect prime ideals in polynomial rings, Arch. Math. 93 (2009), 213-217.

16. V. Erdoğdu, Efficient generation of prime ideals in polynomial rings up to radical, Comm. Algebra 38 (2010), 1802-1807.

17. V. Erdoğdu and S. Harman, Commutative rings whose prime ideals are radically perfect, J. Commutative Algebra 5 (2013), 527-544.

18. M. Fontana, S. Gabelli and E.G. Houston, UMT-domains and domains with Prüfer integral closure, Comm. Algebra 26 (1998), 1017-1039. 
19. M. Fontana, J. Hucaba and I. Papick, Prüfer domains, Marcel Dekker, New York, 1997.

20. R.M. Fossum, The divisor class group of a Krull domain, Springer, New York, 1973.

21. S. Gabelli, On divisorial ideals in polynomial rings over Mori domains, Comm. Algebra 15 (1989), 2349-2370.

22. S. Gabelli, E.G. Houston and G. Picozza, w-divisoriality in polynomial rings, Comm. Algebra 37 (2009), 1117-1127.

23. R. Gilmer, Multiplicative ideal theory, Marcel Dekker, New York, 1972.

24. R. Gilmer and W. Heinzer, Primary ideals with finitely generated radical in a commutative ring, Manuscr. Math. 78 (1993), 201-221.

25. E.G. Houston and M. Zafrullah, On t-invertibility, II, Comm. Algebra 17 (1989), 1955-1969.

26. I. Kaplansky, Commutative rings, Polygonal Publishing House, Washington, 1994.

27. H. Kim, Overrings of t-coprimely packed domains, J. Korean Math. Soc. 48 (2011), 191-205.

28. M.B. Martin and M. Zafrullah, t-linked overrings of Noetherian weakly factorial domains, Proc. Amer. Math. Soc. 115 (1992), 601-604.

29. H. Matsumura, Commutative ring theory, Cambridge University Press, Cambridge, 1986.

30. A. Mimouni, Radically perfect prime ideals in polynomial rings over Prüfer and pullback rings, Comm. Algebra 41 (2013), 1377-1385.

31. S. Oda, Radically principal and almost factorial, Bull. Fac. Sci. Ibaraki Univ. 26 (1994), 17-24.

32. F. Wang and R.L. McCasland, On w-modules over strong Mori domains, Comm. Algebra 25 (1997), 1285-1306.

33. _ On strong Mori domains, J. Pure Appl. Algebra 135 (1999), 155165.

34. M. Zafrullah, A general theory of almost factoriality, Manuscr. Math. 51 (1985), 29-62.

35. Well behaved prime t-ideals, J. Pure Appl. Algebra 65 (1990), 199207.

Incheon National University, Department of Mathematics Education, InCHEON 22012, KOREA

Email address: whan@inu.ac.kr

Hoseo University, School of Computer and Information Engineering, Asan 31499, Korea

Email address: hkkim@hoseo.edu 